LINGUACULTURE, 2, 2012

\title{
IF I HAD HAVE BEEN... ON THE WEB
}

\author{
Nadina Cehan \\ "Babeş-Bolyai" University, Cluj \\ AnCa Cehan \\ Alexandru Ioan Cuza University of Iaşi
}

\begin{abstract}
The paper looks at the nonstandard, so called 'plupluperfect', construction If [Subject] had have [past particple]. Based on inquiries in WebCorp and the BNC and COCA, the paper shows the relative incidence of this construction in the available corpora. The investigation concludes that the construction is more likely to be found in Internet texts and communications. This prompts a brief discussion on the general nature of Internet language and its main characteristics. Moreover, taking into consideration the morpho-syntactic make-up of the construction, certain theories about its occurrence are presented. It is also noted that the construction is by no means new, having been attested centuries ago. Thus, the paper tries to tie in history with present-day trends in language and linguistics.
\end{abstract}

Keywords: plupluperfect, corpus linguistics, web language, nonstandard forms, spoken vs. written English

\section{Setting the scene}

The purpose of this paper is to shed light on a somewhat ignored vernacular English grammatical construction whose history, as it will later transpire, is long and venerable. To investigate the nature and incidence of the additional have in third-conditionals is not an easy feat. Most people have not heard of it, or noticed it.The type of clause in which it appears is so restricted in use as to make it almost invisible. Nevertheless, by looking back at the work of standard English sticklers from the beginning of the $20^{\text {th }}$ century and by using the latest linguistic investigative tools, it becomes possible to thoroughly analyse this unusual occurrence of a superfluous have.

Literature on the construction in question is rather scarce and anecdotal. It is, however, mentioned by two of the most influential defenders 
of the English standard language, H. W. Fowler and Eric Partridge. Their works first appeared in 1926 and 1942, respectively. To understand the perceived status of the extra have one need only look at what these authors have to say about it. Thus, Fowler informs the reader, under the entry "had", that:

"[writing had...have where had is required] has proved fatal in Had she have done it for the Catholic Church, she would doubtless have been canonized as St. Angela; and in Had I have been in England on Monday, I should certainly have been present at the first performance. This is no better than an illiterate blunder, and easily shown to be absurd. Had she, had I, are the inverted equivalents of if she had, if I had; no one would defend If she had have done, nor if I had have been, and it follows that Had she done, Had I been, are the only correct inverted conditionals." $(1965,35)$

Inspired by his predecessor's work, Partridge makes it clear that the "blunder" also occurrs in written texts and notes the existence of yet another nonstandard form found in the same type of conditional:

"had have ('If you had have come'). Redundant have; an error by no means confined to the illiterate. This construction, in which the have is intrusive and which has the still more illiterate variant had of, is not an error I should have signalized here, had it not been for the following sentence met with in a very good novelist, 'But then, thought Rome [an educated woman], should I have been any more understanding if I hadn't have happened to have been there that afternoon when Mark's name was mentioned?"' $(1973,142)$

As the above quotations suggest, the presence of an additional have in the third-conditional subclause is unacceptable and considered ill-educated usage. Although no explanation is offered as to why some speakers feel the need to include it, the texts emphasize its social status. They serve to document its presence both in inverted conditionals and fully-fledged conditional subclauses, i.e. after if. They also imply that nonstandard features are proscribed in "serious public communication, especially in written communication" (Biber et al 1999, 1121), thus indicating that discussions of standard versus nonstandard language are generally the domain of sociolinguistics. In what follows we attempt to assess the relative spread of this nonstandard construction and to potentially explain its survival.

\section{Grammar Talk}

For reasons of brevity and ease of understanding, we will henceforth refer to the nonstandard third-conditional construction under discussion as if [Su] 
had have $+p p$, where [Su] refers to the syntactic category of subject, and is perceived as a slot where any noun phrase could be introduced to fulfil the said function. In addition, $p p$ stands for past participle, also called the third form of the verb. Finally, it is important to note that if [Su] had have + pp here is understood to cover all possible variations of the construction, including inversion, negation, active and passive voice, and all possible combinations thereof.

The syntactic context of if [Su] had have $+p p$ contains two semantic and grammatical features: the perfect and modality. Thus, the construction evinces the unusual double presence of have (one past and one present form), which in English is used to form perfect tense forms (cf. Declerck 2006, 19). As an auxiliary, have has little lexical meaning and is employed in forming what Declerck terms "complex verb forms" (idem). For instance, in standard type three conditionals, as in If I had known, I would have told you, have is present in the subclause where it is backshifted to the past and the past perfect forms, while in the main clause it forms the perfect conditional with would (i.e. would have told you). Except in the case of 'code' (or ellipsis), have is necessarily followed by the past participle of the main verb. Together they form what is sometimes termed the perfect infinitive. As an auxiliary, have evinces the 'NICE properties' that it shares with all other auxiliaries, i.e. it does not require $d o$-support in the cases of negation, inversion, code or emphasis (cf. Declerck 2006, 20). Have and its past counterpart had also allow for contraction: 've and ' $d$.

Perfect verb forms are found in complex constructions which express an anterior temporal relation "between the time of the situation referred to" (Declerck 2006, 25) and the time of a situation which serves as a temporal anchor point. For the (present) perfect the situation is anchored in the present, for the past perfect (also called pluperfect) it is placed in the past, hence the characterization of the temporal relation as being past-in-the-past, while for the future perfect, will anchors the relationship in the future. Because the "main function of this category [i.e. the perfect] is to establish a relationship of anteriority between a certain situation and a point of reference" (Kortmann 2005, 146) other than the utterance time, the perfect is considered by some authors a(n) (absolute-)relative tense (cf. Declerck 2006, Kortmann 2005). However, its exact status is unclear and some argue for its treatment as a separate category, neither tense, nor aspect (cf. Comrie 1985, Kortmann 2005).

The most salient properties of the perfect are that semantically it seems to express the idea of "current relevance" (Comrie 1985, 14), which the pluperfect (i.e. past perfect) loses, and that the past situation is unspecifically determined. More to the point, "the perfect in English [...] cannot collocate with a time adverbial referring to a specific time point in 
the past" (Comrie 1985, 78), that is, a sentence such as *I have finished yesterday is ungrammatical. Nevertheless, as Comrie points out (idem, 789), a sentence such as I have finished at three o'clock is possible when the context clearly allows for a habitual interpretation, so that the act of finishing at that particular time has happened at least once before, at some indeterminate date. Moreover, it is possible for the (present) perfect to allow for collocation with adverbials which have present time reference, indicating the recency of the event, as in I have just/ recently/ now finished the book. Despite the several solid arguments against it, it seems more likely that the perfect is a tense at its core. Although tenses are said to be deictic in the sense of pointing to a past, present or future situation in relation to the moment of utterance, it is possible that the "deictic center" (Comrie 1985, 17) need not necessarily be the present moment, specifically the moment of the utterance, which would allow for the perfect to be interpreted as tense, rather than aspect or an altogether different category.

In terms of modality, conditionals of the third type express counterfactuality or an unprovable hypothesis. In Quirk's phrasing, such contexts convey "the speaker's belief that the condition [...] was not fulfilled $[\ldots]$ and hence the $[\ldots]$ certain falsity of the proposition expressed by the matrix clause" (Quirk et al. 1985, 1091). Thus, type three conditionals are often characterised as being closed or half-closed (cf. Bailey 1989). In a sentence such as If I had known, I would be home by now, where there is a type two conditional (in the main clause) combined with type three (in the subclause), the if-clause expresses the counterfactuality of the proposition backshifted in the past. In the main clause, the use of would + infinitive is the expression of the speaker's certainty in the probable post-present result of the situation expressed in the conditional subclause.

Biber et al. $(1999,837)$ note that during conversations people often tell stories, so that the initial if-clause has the function of setting up a time frame that influences the interpretation of time relationships and references in the subclause and the main clauses. This is a salient observation, since the probable consequences of the proposition raised in an if-clause that conveys a hypothetical condition are discussed in the main clause. Moreover, the same authors point out that the "difference in tense and modality is essential in hypothetical conditional clauses" (idem, 828). The modality present in conditional clauses is on the one hand an expression of the speaker's certainty in the probable or possible outcomes of the proposed condition, and on the other hand, it expresses the unreality of that condition.

\section{Standard and nonstandard, written and spoken English}

Nonstandard constructions such as if [Su] had have $+p p$ are perceived to indicate lack of education and they are usually not even mentioned to 
learners of English as a second or foreign language, since only standard English serves as the model. In their manifestations, non-standard forms are not confined to either the spoken or written registers of the language, as they include lexical, morpho-syntactic, semantic and spelling phenomena. Moreover, as Biber et al. explain:

"vernacular features of grammar can be highly prized because of their role in establishing and maintaining social solidarity among the speakers in selected groups, and in bringing vigour and colour into speech style.” $(1999,1121)$

Furthermore, the distinction between standard and non-standard English is not as clear-cut as it may seem. In actual usage, there is "a continuous range of acceptability" (idem). Finally, some non-standard forms may be found all over the English-speaking world and are not necessarily confined to a regional dialect.

Traditionally, spoken and written English are thought of as two separate entities. Spoken English can occur in a variety of situations, ranging from lectures, sermons, staff meetings to having dinner with one's family. Nevertheless, face-to-face conversation is the most basic context for any spoken variety and it has a number of important features. These include: being "directly interactive [...], involv[ing] a focus on the lives and interests of the interlocutors, [...] [and their] shar[ing] the same physical and temporal context" (Biber et al. 1999, 16). In addition to these, Miller and Weinert identify several other properties of spoken language, including that "[i]nformation is carefully staged, a small quantity of information being assigned to each phrase and clause" and that "[a] number of constructions occur in spontaneous spoken language but not in written language, and viceversa" $(1998,22-3)$. In other words, the nonstandard is mostly regarded as being the domain of the spoken, or, as Leech observes, in speaking people employ "a vernacular range of expression" (Leech 1998).

Written language appears in registers that differ greatly from those generated by conversational situations. On the whole, texts are "not directly interactive [...] and have communicative purposes not focused on the personal concerns of the writer/reader" (Biber et al. 1999, 16). Although written texts usually lack in having a specific addressee, their authors always have a more or less particular audience in mind and are thus writing to accommodate their expectations and preferences, linguistic or content-wise. In addition, from a stylistic point of view, written language is also characterized in terms of distinctive graphic, orthographic, grammatical, lexical and discourse features. Miller and Weinert note that, in general, written and spoken language "cannot be clearly differentiated" $(1998,21)$ as speakers sometimes use grammar and vocabulary features typical of written 
language, but the extent of this phenomenon is largely determined by their "exposure to higher education" (ibidem) and the situational context.

Recent studies on written language have noted that there is a perceivable stylistic shift towards incorporating features of the spoken register. Although this phenomenon is quite limited in its scope, the 'colloquialization' (cf. Mair 2006, 187) of written language is a noteworthy change. It is defined as a shift in language that moves

away from a written norm which is elaborated to maximal distance from speech and towards a written norm that is closer to spoken usage, and away from a written norm which cultivates formality towards a norm which is tolerant of informality and even allows for anti-formality as a rhetorical strategy (idem).

Spoken and written English, on the one hand, and nonstandard and standard English on the other, are frequently defined and discussed in contrast with one another, and they can each be regarded as different varieties of English. Crystal defines variety as "a system of linguistic expression whose use is governed by situational factors" $(2006,6)$, and regards it as being more or less "systematic and predictable" (idem, 7). A similar view is taken by Biber et al., although the term used is register, whose "distinctions are defined in non-linguistic terms, with respect to situational characteristics such as mode, interactiveness, domain, communicative purpose, and topic" (1999, 15). Given such a broad definition of variety, it makes sense to speak about spoken versus written, standard versus non-standard, national and learner varieties, each as a variety of English in its own right. However, it is worth noting that, regardless of the situation one is looking at, varieties interact with each other on all levels. In other words, "[t]he situational characteristics that define [varieties] have direct functional correlates" (Biber et al. 1999, 15). More to the point, features of one variety may come into contrast with those of another, or it may be the case that lexical, grammatical or discursive characteristics of a certain variety may influence another and be adopted by the latter. Therefore, it is not surprising that nonstandard features are usually linked to the spoken register, while the standard is thought to be mainly reinforced and fostered in the written medium.

\section{A corpus analysis of if [Su] had have $+p p$}

For the purposes of this paper, three corpora were analysed in detail: WebCorp, the BNC (British National Corpus) and COCA (Corpus of 
Contemporary American) ${ }^{1}$. For WebCorp, the settings were the following: Google was the search engine, the concordance span was set to twenty words left and right, and the case options were set to 'Case Insensitive'2. For the BNC and COCA, the implicit settings were not changed.

In order to locate the construction under study in the corpora, the inquiries involved departing from the skeletal construction if [Su] had have, where the $[\mathrm{Su}]$ component was subsequently replaced by all the personal pronouns: I, you, he, she, we, they. In the case of WebCorp, the resulting data were first compiled in an Excel file with the complete context, which was allocated an approximate web text type. The full web address was also recorded. Nevertheless, it should be noted that even at this incipient stage certain results generated by WebCorp were not included in the compilation. These were: instances where the structure was under discussion on an ESL or general English usage forum; when the author was clearly non-native (either admittedly so or because the context showed a large amount of grammar and spelling mistakes); lyrics and poems because they arguably fall under 'poetic license' and because one can never be sure whether the person who transcribed the lyrics was a native speaker of English; other irrelevant results, mainly the ones in which the if [Su] had have was not followed by a past participle form of a verb.

In order to check for the construction's overall behaviour, WebCorp, the BNC and COCA were further analysed. For subject-verb inversion, the construction was adapted to had [Su] have and had [Su] not have. These inquiries generated numerous irrelevant instances. For negation, the corpora were tested for the presence of if [Su] had not / n't + have not / n't and the inverted had not / n't [Su] + have not /n't. As regards contraction, inquiries were made as to the existence of if [Su] had + 've (+ n't) and had [Su] 've $(+n ' t)$. Contracted had (' $d)$ was not included in the searches because there is yet another nonstandard third-conditional form, which co-exists with the one under scrutiny, and which uses modal would in the conditional subclause, as in If I would have known, I would have told you. Therefore, it would have

${ }^{1}$ It must be noted that WebCorp, COCA and the BNC were not the only corpora investigated. Since WebCorp showed that the construction under study is found on websites on servers from all over the English-speaking world including New Zealand, Ireland, Canada and Australia, a need was felt to make inquiries in other corpora, such as the ICE (International Corpus of English) corpora and the Santa Barbara Corpus. However, because these further investigations yielded no results, a decision was made to delimit subsequent inquiries dealing with negation and inversion to the first main three corpora.

${ }^{2}$ Please note that this paper is based on research carried out in 2011. Since then, both WebCorp and the Web have changed in ways which may prove considerable if the same inquiries were made at the time when the reader peruses this article. 
been impossible to determine whether the ' $d$ initially meant would or had in the mind of the writer. The results showed that a number of combinations were not possible at all, and they are left out of the final results, which are shown in the following table:

\begin{tabular}{|l|c|c|c|c|}
\hline Construction & WebCorp & COCA & BNC & TOTALS \\
\hline If $[\mathrm{Su}]$ had have + pp & 107 & 2 & 1 & 110 \\
\hline $\begin{array}{l}\text { If }[\mathrm{Su}] \text { had not have } \\
+ \text { pp }\end{array}$ & 153 & 0 & 0 & 153 \\
\hline $\begin{array}{l}\text { If }[\mathrm{Su}] \text { hadn't have }+ \\
\text { pp }\end{array}$ & 236 & 17 & 16 & 269 \\
\hline Had [Su] have + pp & 126 & 3 & 1 & 130 \\
\hline $\begin{array}{l}\text { Had }[\mathrm{Su}] \text { have not }+ \\
\text { pp }\end{array}$ & 40 & 0 & 0 & 40 \\
\hline If $[\mathrm{Su}]$ had've + pp & 204 & 1 & 4 & 209 \\
\hline $\begin{array}{l}\text { If }[\mathrm{Su}] \text { had've not }+ \\
\text { pp }\end{array}$ & 1 & 0 & 0 & 1 \\
\hline TOTALS & 867 & 23 & 22 & 912 \\
\hline
\end{tabular}

Table. Incidence of if [Su] had have $+p p$ and its variants showing negation, inversion and contraction possibilities in WebCorp, COCA and the BNC corpora.

In addition to the hard data, the retrieved results show that the nonstandard construction in question behaves just like a standard one would. Thus, the non-standard past perfect had have + pp collocates not only with if, but also with as if and even if, which are compound conjunctions that introduce unreal conditional clauses, for instance in the following:

By the time he finished rebuilding and replacing the engine, it cost twice as much as if I had have just taken the car to a certified mechanic in the first place!

[http://www.kevindedmon.com/2009/08/yada-yada-yada/, WebCorp]

Hindsight is a wonderful thing and even if he had have called the police, there is no gaurantee that Ethan may still be alive! [http://fatherhood.about.com/b/2010/05/16/the-ethan-stacy-tragedy.htm, WebCorp]

Moreover, it becomes apparent that the construction in question can combine with the progressive aspect, can undergo passive voice 
modification, is used with any type of verb or collocation, and is subject to general syntactic devices such as ellipsis and subject-verb inversion:

If you had have been following the law yourself, you wouldn't have been on the road with no insurance... [http://www.answerbag.com/q_view/2421932, WebCorp]

Even if he had have been sent off Carragher should have had two penalties awarded against him by that point... [http://www.guardian.co.uk/football/2010/sep/19/manchester-united-

liverpool-premier-league, WebCorp]

And, Your Honor, because if you - if I had have been, you would have been calling me on it [1995, SPOK, CNN News, COCA]

If he had have previously self-harmed or shown signs that he would selfharm, he would have been monitored. [http://www.thewestmorlandgazette.co.uk/news/cumbria/4875645.Lady_in_t he_Lake_row_goes_beyond_the_grave/, WebCorp]

In conclusion, using WebCorp to analyse if [Su] had have + pp has revealed two important points. First, the difference in the amount of results retrieved by WebCorp as compared to the other two digital corpora, COCA and the $\mathrm{BNC}$, is quite astounding. On the one hand, it indicates that the latter are somewhat limited in scope and would have not provided sufficient data had they been used alone. On the other hand, the large amount of language that has accumulated on the Web provides the means to look at if [Su] had have $+p p$ in depth. It may also lead to the idea that, given the high incidence of this nonstandard construction, and possibly of others as well, Web language may be thought of as coming closer to the benchmark of spoken English than other written media. However, this discussion remains outside the scope of the present article. The second point is the if [Su] had have + pp nonstandard construction has clearly become entrenched in the language, as it is used unreservedly with all kinds of verbs and follows all applicable syntactic rules.

\section{A construction with a history}

Commentators on if [Su] had have $+p p$ have traced it back to the fifteenth century (cf. Christophersen 1986). Because it is primarily a feature of the spoken register, there is some confusion as to how written instances such as if [Su] had $\boldsymbol{a}+p p$ or if [Su] had of $+p p$ should be analysed. Specifically, whether $a$ or of evince a phonetic writing of have is questionable. Nevertheless, the construction is by no means confined to spoken language, a fact supported by historical evidence. Inquiries in WebCorp have returned 
examples of the construction in digitalised personal letters from the American Civil War. It should be noted that these letters have different authors.

I would have been mighty proud if you had have let Frank come to see me. [http://georgiainfo.galileo.usg.edu/CivilWar/apr464.htm, WebCorp]

But I would have throwed them away if I had have had to have went naked 'till Christmas rather than been captured... [http://georgiainfo.galileo.usg.edu/tdgh-jul/jul02.htm, WebCorp]

\section{A brief discussion of if [Su] had have $+p p$}

The presence of the additional have in the nonstandard if [Su] had have + pp has been given several possible explanations. In short, it may be analysed as a perfect subjunctive completing the conditional subclause paradigm alongside the past subjunctive and the unmarked present subjunctive whose form is identical with the indicative form of the verb. Have could also be accounted for in terms of a phonologic parallel between the conditional subclause and the main clause. Finally, it has been proposed that the function of have is to stress the past-in-the-past time relationship which is already given by the past perfect auxiliary had. It is these authors' opinion that none of these analyses are satisfactory enough and a competing interpretation is argued for.

Earlier discussions of the construction set forth the idea that if [Su] had have $+p p$ could be analysed as a 'perfect subjunctive'. This assessment might be possible in principle because, when looking at the canonical patterns of the conditionals, one finds the past subjunctive present in ifclauses of the second type, as in: If I were you, I wouldn't go, where the verb $b e$ is in the subjunctive mood (as opposed to was, the indicative past simple form, which is more frequent in colloquial English and is gaining ground). However, it must be noted that in irrealis (or hypothetical) conditionals of the third type the form of the verb in the sub-clause is never analyzed as a perfect subjunctive. Moreover, the past subjunctive (also called the 'weresubjunctive') is also used in certain contexts, such as after wish (e.g. I wish I were you) or suppose (e.g. Suppose that were the case, what would you do / have done?). If these examples were to be backshifted and referred to a pastin-the-past time frame, expressing anteriority, they would become: I wish(ed) I had been you and Suppose that had been the case, what would you do / have done?

Such cases are not said to contain a form of perfect subjunctive. Palmer, for instance, rejects such an analysis as it would only confuse the issue (cf. Palmer 1986). Furthermore, the method of data collecting for the 
construction in question did not yield any sentences of the above kind. Thus, the following syntactic contexts ?I wish(ed) I had have been you and ?Suppose that had have been the case what would you have done? were outside the scope of this paper's data collection and analysis. In conclusion, an analysis of if $[\mathrm{Su}]$ had have $+p p$ as a perfect subjunctive remains questionable and requires further extensive inquiries in the available corpora.

A second analysis of the construction proposed that the presence of have can be explained in terms of 'stress and rhythm' (cf. Lambert 1986). As this linguist explains: '[T]he two clauses are parallel [...] (as grammatically one clause contains 'had have' and the other 'would have'), which is [...] the reason why the form evolved in the first place.' (idem, 30) Although this explanation has well-grounded arguments, it is questionable whether phonologic phenomena are strong enough to generate full-fledged nonstandards. Although this may have contributed to the continued presence and survival of the vernacular construction under study, it is the present authors' opinion that there must have been other reasons that have contributed to its development. More to the point, the superfluous presence of have may indicate that this auxiliary brings additional needed semantic undertones to the proposition expressed by the conditional subclause.

Most linguists see in if [Su] had have + $p p$ an instance of emphasis of the past relationship which is already introduced by had (cf. Declerk 2006). This interpretation is sustained by comparing this nonstandard construction with the equally vernacular if [Su] would have $+p p$ and with the standard if [Su] past modal have $+p p$ (i.e. if [Su] could have $+p p$ or if [Su] should have $+p p)$. As Palmer notes on the parallel with the standard forms, '[t]here is a problem with past unreal because it needs to mark past tense twice, once for time and once for unreality' (1986: 30). In other words, past tense forms, especially modal auxiliaries, are capable of carrying both modality and tense. This is also the case of many verbal phrases, including the standard past subjunctive, the standard third type conditional subclause (where the auxiliary had carries the weight of both the irrealis and the anterior past time frame) and even of the nonstandard third conditional expemplified by If I knew, I would have told you.

Notwithstanding all of the above, if a parallel is drawn between the two nonstandard constructions if [Su] had have $+p p$ and if [Su] would have $+p p$, a closer look may suggest another, complementing interpretation. Thus, shifting attention on the past form had in the construction reveals that it behaves similarly to a modal auxiliary: it moves to the front of the clause in case of inversion and it is had (not have) that is usually negated in the construction. In other words, as in the case of would in if [Su] would have + $p p$, or in the apodosis of the standard third conditional (If I had known, I wouldn't have told you), it is modality, the hypothesis, the irrealis, which is 
negated, and not the instantiation of the situation described in the verb phrase.

Moreover, typological considerations also indicate that an interpretation of had in if [Su] had have $+p p$ as modal is possible. English dialects and languages of the world show that some grammars allow for semantic features to recur at morpho-syntactic level. For instance, some regional English dialects allow for double (or multiple) negation, as in: $I$ didn't have no dinner (Hughes 1996, 22). Furthermore, the Romanian language allows for the same past conditional tense to be used in both the protasis and the apodosis: Dacă aş fi ştiut, ţi-aş fi spus, thus repeating the modality.

Given that if [Su] had have + pp surfaces quite frequently on the Web, a few words about this phenomenon may be in order. First, WebCorp is a tool that allows for the Web to be analysed as a traditional electronic corpus, as it combines the capabilities of a Web search engine with those of a standard concordancer. It is used chiefly to investigate rare and very new linguistic phenomena because the Web represents the largest and most recent corpus available, despite its many drawbacks. Second, it should be noted that the results retrieved by WebCorp are not to be taken to be representative of all language that is produced via the Internet or on the Web. All language retrieved by WebCorp is asynchronous and is written for a wide audience (in fact, for anyone who accesses the website). Moreover, WebCorp cannot access language that has been transmitted over e-mail, instant messaging or certain social networks, such as Facebook. These observations bring us to the conclusion that although the Web is an integral part of the Internet, which is "a predominantly written medium" (Crystal 2006, 19), one cannot make generalisations about the overall character of the Web language. In other words, although it would in principle be appealing to say that Web language evinces a further step in the colloqualisation of written language, because it allows for vernacular forms to appear more frequently, this is probably true for only certain genres, or text-types, that have appeared since the Web has come into being.

\section{Conclusions}

The focus of this paper has been the vernacular, nonstandard construction if [Su] had have + pp which was analyzed using corpus techniques. It discussed its formal characteristics, its internal structure, its semantics and its history. Some observations on the nature of spoken and written language were needed in order to justify the use of the Web, and implicitly WebCorp, to better understand the construction's makeup and spread in the English language. The paper has also shown the different interpretations that the construction has received from scholars and the authors have also proposed a 
new possible analysis in view of how the construction has been shown to behave syntactically.

At the end of this journey, more questions seem to impose themselves and thus more avenues for research open up. Perhaps the most relevant question regards the apparent 'fuzziness' of language. Although it is easy and cognitively necessary to think about language in terms of contrasts and hard boundaries: standard vs nonstandard, written vs. spoken, the closed class of modal auxiliary verbs, what the analysis of if [Su] had have $+p p$ indicates is that such limits do not truly exist. It is relatively easy to argue that sometimes language that is spoken, such as in the case of academic presentations, evinces numerous characteristics that are usually associated with the written medium and it is probably clear to most users of the Internet and the Web that written rules and conventions have relaxed in this electronic medium. Yet, it may seem almost impossible to acknowledge that similar phenomena happen at the very base of language: its grammar. What this analysis of if [Su] had have + pp reminds us is that language is fluid and allows its elements to interact and borrow meanings and behaviour from one another.

The analysis of nonstandard forms, especially in the verb phrase, may provide new insights into the nature of the English language and perhaps even the direction in which it is changing. Thus, one direction for further research is that of the other nonstandard third conditionals that populate the English language. It would be interesting to find out their relative frequency and in comparing them to see whether they provide some clarifications about the internal mechanism of the language.

Another road not trodden enough is that of the Web and its language. This is a field which has rarely been meticulously analysed from a purely linguistic point of view. At least two possibilities arise: first, to see how much of Web language is spoken-like and which of its features are more written-like in character. Second, and in connection with the first proposition, to classify the miriad of Web-texts that have been and are being produced into several categories, thus opening up the possibility to identify Web-specific genres.

\section{Works Cited}

\section{Primary sources}

$B N C$ (British National Corpus) and COCA (Corpus of Contemporary American English). http://corpus.byu.edu. Web. March 2011.

WebCorp. http://www.webcorp.org.uk/. Web. March 2011. 


\section{Secondary sources}

Bailey, Charles-James T. "Classifying the English Conditionals." American Speech 64, 3 (1989). 275-80. Print.

Biber, Douglas and Stig Johansson, Geoffrey Leech, Susan Conrad, Edward Finegan. Longman Grammar of Spoken and Written English. Harlow: Longman. 1999. Print.

Comrie, Bernard. Aspect. Cambridge: Cambridge University Press. 1976. Print.

Christophersen, Paul. "A history of the plupluperfect." English Today 8, 2 (1986). 36. Print

Crystal, David. A Dictionary of Linguistics and Phonetics. $5^{\text {th }}$ edition. Malden: Blackwell. 2003. Print.

Crystal, David. Language and the Internet. $2^{\text {nd }}$ edition. Cambridge: Cambridge University Press. 2006. Print.

Declerck, Renaat. The Grammar of the English Verb Phrase. Volume 1: The Grammar of the English Tense System. Berlin: Mouton de Gruyter. 2006. Print.

Fowler, H. W. A Dictionary of Modern English Usage. $2^{\text {nd }}$ edition. Revised by Sir Ernest Gowers. London: Oxford University Press. 1965. Print.

Hughes, Arthur and Peter Trudgill. English Accents and Dialects. $3^{\text {rd }}$ edition. London: Arnold. 1996. Print

Kortmann, Bernd. English Linguistics: Essentials. Berlin: Cornelsen. 2005. Print.

Lambert, Valerie. "The nonstandard third conditional." English Today 8, 2 (1986). 28-9. Print.

Leech,Geoffrey. "English Grammar in Conversation." http://www.tuchemnitz.de/phil/english/chairs/linguist/real/independent/llc/Conference1998/Pa pers/Leech/Leech.htm, 1998. Web. November 2012.

Mair, Christian. Twentieth-Century English: History, Variation and Standardization. Cambridge: CUP. 2006. Print.

Merriam-Webster's Dictionary of English Usage. Massachusetts: Merriam-Webster Incorporated. 1995. Print.

Miller, Jim and Regina Weinert. Spontaneous Spoken Language: Syntax and Discourse. Oxford: Claredon Press. 1998. Print.

Quirk, Randolph, Sidney Greenbaum, Geoffrey Leech, and Jan Svartvik. A comprehensive grammar of the English language. London: Longman. 1985. Print.

Palmer, Frank. "Plupluperfect or pluplupast?" English Today 8, 2 (1986). 29. Print.

Partridge, Eric. Usage and Abusage: A Guide to Good English. London: Penguin Books. 1973. Print

Trudgill, Peter and Jean Hannah. International English: A guide to varieties of Standard English. $4^{\text {th }}$ edition. London: Hodder Arnold. 2002. Print. 